TRANS · núm. $24 \cdot 2020$

MISCELÁNEA · 375-400
El objetivo de esta contribución consiste en demostrar la validez y aplicabilidad de un instrumento de recopilación de datos adecuado para recabar información relativa al uso y la percepción de las herramientas de traducción automática y posedición en el mercado actual de la traducción en España, en este caso, un cuestionario virtual. Con este propósito, se va a contextualizar este estudio mediante un recorrido por aquellos aspectos teóricos relativos a la metodología social y cualitativa que se han considerado más significativos para su configuración. Asimismo, se van a detallar las diferentes subetapas de las que se compuso la fase de elaboración del instrumento utilizado: diseño, pruebas de robustez, lanzamiento, recogida de datos e informe de resultados. En la subetapa de diseño del cuestionario se citarán como antecedentes de este trabajo diferentes estudios que se han considerado relevantes para su realización por su similitud en cuanto a la metodología utilizada. A continuación, en la subetapa de pruebas de robustez, se describirán los pasos seguidos para optimizar el diseño del cuestionario, entre los que se encuentran el panel de jueces y el pilotaje. Seguidamente, se explicará el procedimiento llevado a cabo para el lanzamiento del cuestionario virtual, basado en la técnica de muestreo no probabilístico denominada bola de nieve, así como el método escogido para la recogida de los datos. Por último, se presentarán algunos de los datos más relevantes recopilados relativos al uso y actitud de los traductores profesionales respecto a la traducción automática y a la posedición. Asimismo, se presentarán las principales conclusiones generales que se desprenden del trabajo realizado.

PALABRAS CLAVE: estudio social cualitativo, cuestionario, traducción automática, posedición.

\title{
El uso del cuestionario para conocer la recepción de la traducción automática y la posedición en España
}

\author{
Lorena Pérez Macías \\ Universidad Autónoma \\ de Madrid
}

\section{The Use of the Survey to Find Out About the Perception of Machine Translation and Post-Editing in Spain}

The aim of this contribution is to demonstrate the validity and applicability of an appropriate data collection instrument to collect information on the use and perception of machine translation and post-editing tools in the current Spanish translation market, in this case, a virtual survey. To this end, this study will be contextualised through a review of those theoretical aspects relating to social and qualitative methodology that have been considered most significant for its configuration. Furthermore, the different sub-stages of the development phase of the instrument used will be detailed: design, robustness tests, launch, data collection and reporting of results. In the survey design substage, different studies that have been considered relevant for this work due to their similarity in terms of the methodology used will be cited as background for this work. Next, in the robustness testing sub-stage, the steps taken to optimise the survey design will be described, including the panel of judges and piloting. Then, the procedure carried out to launch the virtual survey, based on the non-probability sampling technique called snowball, will be explained, as well as the method chosen for the data collection. Finally, some of the most relevant data collected on the use and attitude of professional translators with regard to machine translation and post-editing will be presented. The main general conclusions arising from the work carried out will also be presented.

KEY WORDS: qualitative social study, survey, machine translation, post-editing. 


\section{INTRODUCCIÓN}

Hoy en día es innegable que las tecnologías aplicadas habitualmente a los procesos de traducción cada vez integran más funcionalidades próximas a la traducción automática o propias de este tipo de procesos, a pesar de que son muchas voces las que, a lo largo de la historia de la traducción, han tendido a percibir con dudas, reticencia o recelo los avances de la traducción automática, como Nida (1964) o Way (2016) en dos extremos temporales de la historia de esta disciplina. Es por ello que, en un entorno tan cambiante como el del mercado profesional de la traducción, es importante no perder de vista al traductor humano, ya que finalmente es quien debe lidiar diariamente con las novedades que estén a su alcance, por lo que, como agente principal, es primordial conocer cuál es su opinión, actitud, etc. en relación con estos cambios. Con este objetivo, se efectuó un estudio cualitativo de carácter práctico e interpretativo de recopilación de datos en el marco del programa de doctorado Lenguas Modernas, Traducción y Español como Lengua Extranjera de la Universidad Pablo de Olavide (Pérez-Macías, 2017).

Es importante indicar en este punto que la mayoría de los estudios sobre traducción automática y posedición hasta la fecha no se realizan desde un enfoque metodológico sociológico como el que aquí se presenta, sino más bien desde coordenadas tecnológicas, cognitivas o lingüísticas, como es el caso, por ejemplo, de los trabajos de O'Brien, 2002; Koponen, 2010; entre innumerables estudios que están apareciendo de forma constante y prolífica en los últimos años. Asimismo, si limitamos el tema de la investigación social al estudio de las percepciones de los traductores respecto a algún ámbito del sector, no son demasiados los estudios que se han podido tomar como antecedentes de este trabajo, ya que hasta el momento apenas se han realizado investigaciones que coincidan significativamente con los objetivos de este estudio. Entre esos estudios que pueden tener relación parcial con los objetivos y métodos aquí planteados, se detallarán algunos en el apartado 2 del presente trabajo.

Este estudio que aquí se describe tiene como propósito principal demostrar la validez y aplicabilidad del cuestionario como instrumento de recopilación de datos para recabar información relativa a la recepción de las herramientas de traducción automática y posedición en el mercado actual de la traducción en España, así como presentar algunos de los resultados recopilados más relevantes. Para ello, se explicarán en detalle cada una de las diferentes subetapas de las fases de elaboración y aplicación del cuestionario: diseño, pruebas de robustez, lanzamiento, recogida de datos e informe de resultados. Por último, se presentarán, analizarán e interpretarán algunas de las percepciones más relevantes registradas a través del feedback de los 104 sujetos que formaron la muestra del estudio.

\section{LA INVESTIGACIÓN SOCIAL CUALITATIVA APLICADA A LOS ESTUDIOS DE TRADUCCIÓN}

Llegados a este punto, es necesario explicar en detalle a qué hace referencia el término cualitativo, puesto que esta denominación se repite a lo largo de todo este trabajo en numerosas ocasiones y puede designar dos realidades distintas que es posible confundir. Por un lado, puede referirse a los datos no numéricos. Sin embargo, tiene también una acepción más amplia y bien extendida en metodología de la investigación, que se refiere a una manera particular de enfocar una investigación científica cuyo fin no es la cuantificación estadística pura (resultante en la inferencia estadística y la generalización), sino 
la descripción de realidades insuficientemente exploradas (Grotjahn, 1987: 57-58).

Por norma general, las investigaciones enmarcadas dentro del ámbito social se suelen vincular con frecuencia a dicho enfoque cualitativo, si bien pueden existir igualmente estudios sociales de corte experimental. En el ámbito de las investigaciones sobre traducción, con respecto a la diferencia que existe entre metodología cualitativa y metodología cuantitativa, Ana Rojo (2013) señala que la base de un diseño cualitativo reside en analizar la naturaleza de los datos e interpretar sus atributos y esencia, mientras que la de un diseño cuantitativo, por el contrario, radica en medir la cantidad de dichos datos e interpretarla como indicador de su naturaleza. Asimismo, indica la autora, que el investigador cualitativo solo necesita un número reducido de ejemplos para ilustrar la existencia de un determinado fenómeno, mientras que en un estudio de corte cuantitativo es necesario un número significativo de ejemplos para que un fenómeno sea representativo.

Para decidir cuál es el enfoque que un determinado estudio requiere, es necesario tener claro inicialmente qué se quiere estudiar y qué datos se quieren recopilar en dicha investigación, ya que el método de investigación debe adaptarse a la naturaleza del objeto de estudio, al contexto estudiado y a los objetivos de la investigación (Oppenheim, 1992; Silverman, 2000).

De igual manera que ocurre con el término cualitativo, es posible hacer una distinción entre las distintas aplicaciones del concepto interpretativo. Grotjahn (1987: 56-57) indica que puede hacer referencia, por un lado, a un modo concreto de análisis de los datos y, por el otro, a un modelo de investigación completo. Respecto a este último significado, es importante resaltar que es bastante común entre los investigadores asociar conjuntamente los conceptos enfoque cualitativo y enfoque interpretativo, por sus grandes similitudes (González, 2001: 234). No obstante, para otros autores, como Dvora Yanow (2014: xvii), resulta fundamental destacar las particularidades del enfoque interpretativo respecto al cualitativo puro, aunque puedan aparecer de manera combinada en muchos casos. La autora señala respecto a esta cuestión:

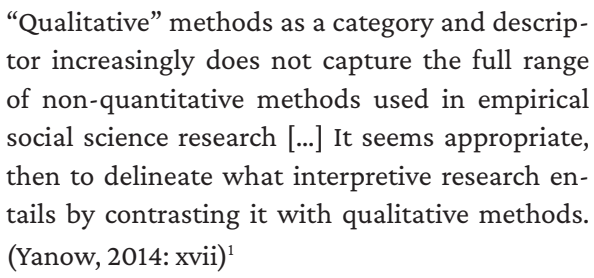

En cuanto a la metodología interpretativa, Calvo (2009: 329) puntualiza en su tesis doctoral que, en este enfoque, la comprensión se basa en la relación que desarrolla el informante entre los aspectos estudiados apoyándose «en sus propias reglas de regularidad, reconstruyéndose así la visión que tiene del mundo y de sí mismo, su conocimiento cotidiano».

Respecto al investigador interpretativo y los datos recopilados en un estudio de este tipo, Michael Bassey (2005: 38) explica lo siguiente:

To the interpretive researcher, the purpose of research is to describe and interpret the phenomena of the world in attempts to get shared meaning with others [...] The data collected by interpretive researchers is usually verbal [...] (Bassey, 2005: 38) ${ }^{2}$

I [ES] Cada vez más, los métodos «cualitativos» como la categoría y el descriptor no consiguen captar la gama completa de métodos no cuantitativos utilizados en la investigación empírica en las ciencias sociales [...] Parece apropiado, entonces, delimitar lo que implica la investigación interpretativa contrastándola con los métodos cualitativos. [Traducción propia].

2 [ES] Para el investigador interpretativo, el propósito de la investigación es describir e interpretar los fenómenos del mundo en un intento de obtener un significado compartido con otros [...] Los datos recopilados por los investigadores interpretativos suelen ser orales. [Traducción propia]. 
378 El autor refleja, asimismo, la complejidad de la investigación interpretativa, ya que dependiendo de las desigualdades que se den en la percepción y en la interpretación de la realidad que se pretenda estudiar, junto con las diferencias que puedan existir entre lenguas para designar un mismo concepto, pueden surgir percepciones muy dispares respecto al objeto de estudio por parte de cada sujeto de la muestra (Bassey, 2005: 38).

En cuanto al procedimiento de recopilación de datos en la investigación social, aunque son numerosos los métodos que existen, se prestará una mayor atención al cuestionario por ser el instrumento principal en este estudio. AnderEgg (1995) explica que, normalmente, bajo la denominación de métodos de encuesta se agrupan tanto la entrevista como el cuestionario. Ambos métodos se diferencian, a grandes rasgos, en que en la entrevista los sujetos responden de manera verbal y es necesaria la figura del entrevistador, mientras que, en el cuestionario, las respuestas se ofrecen por escrito y no es necesario que nadie guíe el procedimiento (Ander-Egg, 1995: 245). De igual modo, el autor indica otros métodos de recopilación de datos como la observación, la recopilación documental y el análisis de contenido, que no se van a recoger con mayor profundidad en este estudio puesto que no resultan relevantes para la contextualización de este trabajo. Por otro lado, y más recientemente, Kuznik et al. (2010) se centran en el uso de la encuesta específicamente en los estudios relacionados con el ámbito de la traducción. No obstante, es importante indicar que aquí el término encuesta se utiliza únicamente para referirse al método del cuestionario. Junto a este, las autoras indican otros tres métodos de recopilación de datos que suelen ser frecuentes en la investigación relacionada con la Traductología: la entrevista, el grupo de discusión y la observación directa (Kuznik et al., 2010: 317). La elección de la encuesta como método en los estudios dentro del ámbito de la traducción se realiza cuando el propósito que se pretende conseguir es estratégico o exploratorio, como ocurriría en el estudio que se va a presentar aquí, dado que con este instrumento es posible conseguir datos de manera amplia y general (Kuznik et al., 2010: 342).

\subsection{Antecedentes de estudios sociales basados en cuestionarios}

Una vez explicados todos estos conceptos relativos a la investigación social, a continuación, se presenta una enumeración de estudios de tipo social pertenecientes a la rama de Traductología que utilizan el cuestionario como método de recopilación de datos. Estos estudios, en la mayoría de los casos, han servido como punto de partida e inspiración en la elaboración del cuestionario de este estudio. Es evidente que no es posible mostrar en este listado todos los estudios que cumplen las características mencionadas, por lo que se van a presentar únicamente los que se consideren más relevantes en el marco de este trabajo. Como punto de partida para la realización de esta enumeración, se tendrán en cuenta los trabajos incluidos en el bloque denominado Encuestas orientadas a mejorar la adecuación entre la formación y el mercado laboral, en la recopilación presentada por Kuznik et al. (2010: 324-332) y, a partir de ahí, se añadirán otros estudios similares más recientes. Para facilitar la visualización de todos los datos, se van a presentar en una tabla de manera resumida los aspectos principales de cada estudio, para luego pasar a describirlos más detalladamente:

El primer estudio que se ha incluido en esta recopilación es el de Golden et al. (1992), estudio 
Tabla 1. Recopilación de estudios de tipo social con encuesta en Traductología (Fuente: elaboración propia)

\begin{tabular}{|c|c|c|c|}
\hline AUTOR/ES & TEMÁTICA ESTUDIO & POBLACIÓN & MUESTRA \\
\hline $\begin{array}{l}\text { Golden, Hurtado } \\
\text { y Piqué (1992) }\end{array}$ & $\begin{array}{l}\text { Situación de la TeI en } \\
\text { Cataluña. }\end{array}$ & $\begin{array}{l}\text { 1) Empleadores de los } \\
\text { traductores en Cataluña. } \\
\text { 2) Representantes de la oferta } \\
\text { laboral en TeI en Cataluña. }\end{array}$ & $\begin{array}{l}\text { Muestreo no probabilístico. } \\
\text { Baja participación. } \\
\text { 1y 2) N/d. }\end{array}$ \\
\hline $\begin{array}{l}\text { Mackenzie } \\
(2000)\end{array}$ & $\begin{array}{l}\text { Competencias relativas a la } \\
\text { TeI en Finlandia. }\end{array}$ & $\begin{array}{l}\text { 1) Usuarios de servicios de } \\
\text { traducción en Finlandia. } \\
\text { 2) Proveedores de servicios de } \\
\text { traducción en Finlandia. }\end{array}$ & $\begin{array}{l}\text { Muestreo no probabilístico. } \\
\text { 1) } 58 \text { sujetos. } \\
\text { 2) } 149 \text { sujetos. }\end{array}$ \\
\hline $\operatorname{Li}(2000)$ & $\begin{array}{l}\text { Formación en TeI en Hong } \\
\text { Kong. }\end{array}$ & $\begin{array}{l}\text { Traductores profesionales en } \\
\text { Hong Kong. }\end{array}$ & $\begin{array}{l}\text { Muestreo no probabilístico. } \\
\text { Cuestionario: } 42 \text { sujetos. } \\
\text { Entrevista: } 12 \text { sujetos. }\end{array}$ \\
\hline Calvo (2004) & $\begin{array}{l}\text { La interpretación social } \\
\text { en la Administración de la } \\
\text { provincia de Toledo. }\end{array}$ & $\begin{array}{l}\text { Informantes funcionarios } \\
\text { de distintos ámbitos de la } \\
\text { Administración Pública en la } \\
\text { provincia de Toledo. }\end{array}$ & $\begin{array}{l}\text { Muestreo no probabilístico. } \\
60 \text { sujetos. }\end{array}$ \\
\hline $\operatorname{Lim}(2005)$ & $\begin{array}{l}\text { Estudios de interpretación } \\
\text { en Seúl. }\end{array}$ & $\begin{array}{l}\text { Estudiantes de interpretación } \\
\text { coreano-inglés en Seúl. }\end{array}$ & $\begin{array}{l}\text { Muestreo no probabilístico. } \\
88 \text { sujetos. }\end{array}$ \\
\hline Ortega (2006) & $\begin{array}{l}\text { La interpretación judicial } \\
\text { en el ámbito penal en } \\
\text { España. }\end{array}$ & $\begin{array}{l}\text { Intérpretes judiciales en } \\
\text { plantilla y autónomos. }\end{array}$ & $\begin{array}{l}\text { Muestreo no probabilístico. } \\
\text { Fase previa: } 19 \text { sujetos. } \\
\text { Fase principal: } 64 \text { sujetos. }\end{array}$ \\
\hline $\begin{array}{l}\text { Havumetsä } \\
(2012)\end{array}$ & $\begin{array}{l}\text { El rol del cliente en el acto } \\
\text { de la traducción. }\end{array}$ & $\begin{array}{l}\text { Empresas pertenecientes a la } \\
\text { Finnish-Russian Chamber of } \\
\text { Commerce (FRCC). }\end{array}$ & $\begin{array}{l}\text { Muestreo no probabilístico. } \\
104 \text { sujetos. }\end{array}$ \\
\hline Cerezo (2012) & $\begin{array}{l}\text { Formación universitaria en } \\
\text { traducción audiovisual. }\end{array}$ & $\begin{array}{l}\text { Profesores, traductores y } \\
\text { empleadores. }\end{array}$ & $\begin{array}{l}\text { Muestreo no probabilístico. } \\
\text { Entrevista: } 15 \text { sujetos. } \\
\text { Cuestionario: } 93 \text { sujetos. }\end{array}$ \\
\hline
\end{tabular}

considerado como, probablemente, el primer estudio traductológico en España que incluye una encuesta. Este trabajo formaba parte de un proyecto regional en Cataluña en el que colaboraron la Escola Universitària de Traductors $i$ d'Intèrprets (EUTI), el Institut de Sociolingüística Catalana (ISC) y el Consell Interdepartamental de Recerca i Innovació Tecnològica (CIRIT), y con el que se pretendía describir la situación del mercado de la traducción y la interpreta- ción en Cataluña. Para ello, se delimitaron dos públicos objetivos diferentes: en primer lugar, teniendo en cuenta la posible demanda de servicios de traducción, se dirigió el estudio a potenciales usuarios de servicios de traducción; $y$, más adelante en el tiempo, se prestó atención al otro lado del mercado, a la oferta de servicios de traducción, es decir, a traductores e intérpretes que ejercieran su labor en Cataluña. Para cada uno de esos públicos objetivos se diseñó un 
380

cuestionario cuantitativo específico que se envió a cada sujeto mediante correo postal. La técnica de muestreo fue no probabilística ${ }^{3}$ y el nivel de participación en ambos cuestionarios fue muy bajo (en el primer caso, 3,96\%, y en el segundo, $23,53 \%)$, por lo que el tamaño de la muestra no se considera representativo y los resultados del estudio solo se pueden generalizar al limitado número de sujetos sondeados. No obstante, algunos de los resultados más relevantes obtenidos en este estudio serían: preponderancia de la traducción sobre la interpretación; importante demanda de traducciones con el par de lenguas castellano y catalán, sobre todo de textos literarios; entre otros.

Respecto al estudio llevado a cabo por Rosemary Mackenzie (2000), se trataba de una investigación enmarcada dentro del proyecto POSI (Practical Orientation of Studies in Translation and Interpreting). Este proyecto se desarrolló originalmente en Alemania con el fin de mejorar la equivalencia de las habilidades relativas a la traducción e interpretación en la vida laboral, pero pronto se convirtió en un proyecto de ámbito europeo (Mackenzie 2000: 213). El estudio de Mackenzie se llevó a cabo en la Universidad de Turku (Finlandia) y tenía como objetivo conocer la opinión en torno a las competencias de los traductores e intérpretes en Finlandia de, por un lado, usuarios de servicios de traducción y, por el otro, de proveedores de servicios de traducción. Con este fin se diseñó un mismo cuestionario para ambos públicos objetivos. El muestreo se realizó de manera no probabilística. La muestra sondeada de la primera población (usuarios de servicios de traducción) estuvo formada por

3 Robson (2011: 271) define las muestras probabilísticas como aquellas en las que se conocen las probabilidades de elección de cada unidad muestral, mientras que las no probabilísticas serían aquellas en las que no se conoce esa probabilidad.
58 sujetos en total. En cuanto a la muestra de la segunda población (proveedores de servicios de traducción), estuvo compuesta en total por 149 respuestas (Mackenzie, 2000: 214). Entre los resultados obtenidos en este estudio destacan, por ejemplo, la necesidad de incluir profesores que se dediquen profesionalmente a la traducción o de profundizar en algunos tipos de traducción durante la formación de los futuros profesionales.

El siguiente estudio que se va a detallar es el llevado a cabo en 1998 por Defeng Li (2000) en la Universidad China de Hong Kong. El objetivo principal de este estudio consistía en mejorar la formación en traducción a través del conocimiento de las necesidades que existían en el mercado profesional de la traducción en Hong Kong. El estudio de Li combinaba dos métodos de recopilación de datos: en primer lugar, se envió un cuestionario cuantitativo a traductores profesionales en Hong Kong (mediante una técnica no probabilística de muestreo) que, finalmente, contestaron 42 sujetos; $y$, en segundo lugar, se llevó a cabo una entrevista semiestructurada con el fin de recopilar datos cualitativos con 12 sujetos seleccionados de la muestra anterior. Entre los resultados obtenidos en este estudio destacan: selección del estilo correcto para cada género textual en inglés y en la interpretación como mayor reto de los profesionales; mayor demanda de traducciones de chino a inglés, de traducciones sintéticas y de traducciones relacionadas con China continental; interés por mejorar competencia en literatura y lengua inglesa; entre otros.

Elisa Calvo (2004) lleva a cabo en el año 2000 un estudio con cuestionario sobre la interpretación social, enmarcado en el programa de doctorado Traducción, Sociedad y Comunicación de la Universidad de Granada. El objetivo principal de este estudio era conocer las necesidades y percepciones de los funcionarios de las distintas delega- 
ciones de la Administración Pública en la provincia de Toledo respecto a la interpretación social. La población de este estudio estaba formada por informantes funcionarios de distintos ámbitos de la Administración Pública (Jefatura Superior de Policía, Justicia, Delegación de Educación, entre otros). Los datos se recogieron a través de un cuestionario que se entregaba durante una entrevista individual (en total se realizaron 60 entrevistas). Entre los resultados más significativos obtenidos en este estudio destacan: reconocimiento de la necesidad de contar con el apoyo de intérpretes en el ámbito provincial; existencia generalizada de malentendidos o problemas de comunicación; y desconocimiento del total de la muestra del concepto interpretación social.

El siguiente estudio que se incluye en esta enumeración es el realizado por Hyang-Ok Lim (2005) en la Hankuk University of Foreign Studies en Seúl. El fin de esta investigación era conocer las expectativas, opiniones y necesidades de los alumnos de esta universidad respecto a los estudios que estaban cursando para contribuir en su mejora. Para ello, se envió un cuestionario a estudiantes de interpretación de coreanoinglés de primer y segundo curso. Para llegar a la muestra no se aplicó ninguna técnica probabilística y se obtuvieron 44 respuestas de alumnos del primer curso (79 \% de participación) y otras 44 de los de segundo curso ( $85 \%$ de participación). Como resultado más llamativo, cabe destacar el predominio del género femenino en los estudios de interpretación, ya que esta profesión representa una manera de ascenso social para las mujeres de Corea del Sur (Lim, 2005: 183).

En su tesis doctoral, Ortega (2006), llevó a cabo un estudio descriptivo mediante cuestionario con el objetivo de presentar una panorámica de la interpretación judicial en el ámbito penal y analizar el papel del intérprete. La población objetivo estaba formada por intérpretes ju- diciales, tanto en plantilla como autónomos. El estudio se divide en dos fases diferenciadas: una primera fase, perteneciente a un estudio previo del autor, en la que se recopilaron 19 respuestas; $y$, una fase principal, en la que se obtuvieron 64 respuestas. El método de muestreo fue, nuevamente, no probabilístico. Entre los resultados obtenidos destacan algunos como, por ejemplo, que la mayoría de los encuestados ha adquirido las técnicas de interpretación de manera autodidacta (el $66 \%$ ), mientras que la formación universitaria (con un $42 \%$ ), quedó en segundo lugar, o que casi la mitad de los encuestados considera que, por regla general, les avisan con antelación suficiente de cara a la preparación del encargo, entre otros.

Havumetsä (2012: 95) realizó en su tesis doctoral un estudio con cuestionario en línea en 2006 con el objetivo de explorar y describir las normas que imponen los clientes respecto a la traducción y al traductor y el papel que desempeñan en el acto de la traducción. El cuestionario se envió a 533 empresas pertenecientes a la Finnish-Russian Chamber of Commerce (FRCC) y el método de muestreo fue no probabilístico. La muestra estuvo formada por 104 respuestas. Entre los resultados obtenidos en este estudio destacan: la importancia que le dan los encuestados a que el proceso de traducción se base en la cooperación entre el traductor y el cliente, que esa cooperación se refleje también en el reparto de responsabilidad respecto a la calidad de la traducción o que el traductor debe conocer siempre el propósito de la traducción que se le encomienda.

Por su parte, Beatriz Cerezo (2012), también en su tesis doctoral, lleva a cabo un estudio mediante entrevistas y cuestionarios como instrumentos de recopilación de datos con el fin principal del conocimiento de la situación actual de la formación en traducción audiovisual 
382 para colaborar en la búsqueda de mejoras. La población en este estudio estaba formada por profesores, traductores y empleadores vinculados a este campo de la traducción. En primer lugar, se llevaron a cabo entrevistas semiestructuradas con 5 representantes de cada uno de los grupos de población (5 profesores, 5 traductores y 5 empleadores). Posteriormente, se utilizó un cuestionario en línea distinto para cada población que fue distribuido a través de un método no probabilístico de muestreo. Se recogieron 93 respuestas en total entre todos los tipos de cuestionarios. Algunos datos obtenidos con las respuestas de la muestra sondeada en este estudio serían, por ejemplo, que la traducción audiovisual se imparte en la mayoría de los casos como asignatura independiente y con carácter optativo, que se percibe una escasez de prácticas en el aula que muestren las condiciones reales de trabajo del traductor, que los empleadores consideran necesaria la formación específica en traducción audiovisual, entre otros.

\section{ESTUDIO EMPÍRICO}

Con el fin de conocer la realidad respecto a las percepciones sobre traducción automática y posedición dentro del mercado profesional de la traducción en España, la autora de la contribución que aquí se presenta llevó a cabo un amplio estudio práctico e interpretativo de recopilación de datos en el marco del programa de doctorado Lenguas Modernas, Traducción y Español como Lengua Extranjera de la Universidad Pablo de Olavide (Pérez-Macías, 2017), del que se extraen, a continuación, los siguientes datos.

El público objetivo del estudio estaba formado por traductores en activo en España que conocieran lo que es la posedición de textos generados con traducción automática, tuvieran o no experiencia en ese ámbito. Los criterios de inclusión en el estudio que se establecieron fueron: 1) dedicarse en el momento de participar en el estudio a alguna actividad profesional relacionada con el ámbito de la traducción en España; 2) estar en activo en el momento de participar en el estudio; y 3) considerarse familiarizado con la posedición en términos profesionales. Cabe destacar aquí que, desde el momento de la planificación del estudio, se contemplaba la posibilidad o casi certeza de que la muestra difícilmente podría alcanzar niveles de definición suficientes con respecto al total de profesionales al que va dirigido el estudio, ya que no existe un censo que se pueda consultar donde aparezcan registrados el número total y los datos de contacto de los traductores autónomos en activo en España que además tengan conocimientos de la posedición de textos generados con traducción automática. El único dato que se podría conseguir sería el número de sujetos en activo como autónomos que estuvieran dados de alta en «actividades de traducción e interpretación» (código CNAE 7430), pero tampoco sería de utilidad esa información, puesto que no se podría disponer de los datos de contacto de esos profesionales. Sin embargo, en este estudio, se ha considerado más interesante estudiar a fondo una muestra cualitativamente representativa de la población objetivo, en lugar de aspirar a llegar a un número estadísticamente representativo de profesionales de la traducción que trabajen en España, si se parte de la base, además, de que no sería posible realizar un cálculo estadístico al desconocer la población objetivo total.

\subsection{La triangulación metodológica en la investigación de percepciones}

La primera fase de la parte empírica del estudio antes citado estaba enfocada a la recopilación de datos de naturaleza cualitativa a través de una entrevista grupal previa de aproximación al 
tema, en la que se tuvo un primer contacto con una muestra del público objetivo del estudio y que sirvió para la categorización de los puntos principales que formarían parte de la investigación. Para el diseño de este instrumento, se elaboró un guion semiestructurado que se utilizó durante la entrevista con el fin de orientar la sesión (anexo I). Una vez que tuvo lugar la entrevista, se realizó la transcripción de la información obtenida con ayuda de unos archivos de audio grabados durante la sesión y, posteriormente, se identificaron los nuevos conceptos e ideas que habían surgido para tenerlos en cuenta en la categorización previa que se había realizado e incluirlos en el diseño del cuestionario.

En la segunda fase, dedicada a la recopilación de datos cuantitativos, se diseñó un borrador del cuestionario partiendo de los datos recopilados en la fase previa. A continuación, se va a detallar todo el proceso de diseño y validación del cuestionario, por ser, como ya se ha comentado antes, la herramienta principal de recopilación de datos del estudio que aquí se presenta.

\subsection{El cuestionario}

Una vez analizadas las posibilidades que ofrecería en este estudio la aplicación de una herramienta de recopilación de datos como el cuestionario, se comenzó con la elaboración de este instrumento, fase que comprendió varias subetapas que se extendieron a lo largo del tiempo (desde julio de 2016 a febrero de 2017) y que se explicarán detalladamente a continuación por orden cronológico.

\section{Diseño del cuestionario}

Tal y como indica Hernández (1998: 92), el diseño es la operacionalización (u operativización) de las hipótesis de investigación (aquí, objeto de estudio), es decir, es la transformación de las preguntas y las hipótesis de investigación en las estrategias para: seleccionar a los participantes, aplicar el tratamiento, utilizar los instrumentos de medida, recoger los datos, etc.

Tras la lectura de algunas referencias sobre metodología de cuestionarios en general y el análisis de cuestionarios que ya habían sido utilizados en otros estudios previos tanto dentro como fuera del ámbito de la traducción (se presentará una lista con esos trabajos que han servido para la fundamentación metodológica inicial más adelante), se realizaron varios borradores hasta dar con un modelo aceptable.

Robson (2011: 63) defiende que se debe ampliar la investigación a otros campos y disciplinas distintos al que ocupa el estudio, ya que otros investigadores habrán tenido que lidiar con los mismos problemas que nosotros y los habrán finalmente solucionado. Como se ha visto, en la fase de documentación para este trabajo, se han recorrido diversos estudios basados en cuestionarios con poblaciones diferentes relacionadas con el mundo de la traducción y la interpretación, con el fin de observar de qué forma se han abordado cuestiones compartidas con la presente investigación.

Para crear el modelo que se iba a utilizar en este estudio, se partió del guion creado para la fase de aproximación (entrevista grupal) y se añadieron algunos temas que surgieron a lo largo de la sesión y que se consideraron suficientemente relevantes como para incluirlos en el estudio. Adicionalmente, se modificó la formulación en algunas preguntas (o incluso, en ciertos casos, se eliminaron) para evitar que pudieran resultar confusas o poco eficaces. Algunas recomendaciones que se siguieron para la elaboración del cuestionario fueron las de Fink (1995); Buendía et al. (1998); Wright (2005); y Calvo (2009); que se muestran resumidas en el siguiente listado: 
384

- Incluir instrucciones para la cumplimentación del cuestionario.

- Asegurar confidencialidad en las respuestas.

- Indicar el tiempo necesario para completar el cuestionario.

- Redactar las preguntas de manera que resulten atractivas para el lector y efectivas en la consecución de los objetivos del estudio.

Asimismo, los cuestionarios utilizados en otros estudios recientes dedicados a la recopilación de opiniones, actitudes, perspectivas, etc. ya publicados que se tuvieron en cuenta como fundamentación metodológica previa a la elaboración del diseño de la encuesta fueron: Guerberof (2012); Havumetsä (2012); Cerezo (2012); Pérez-Macías (2013); Alonso (2014); Zaretskaya, Corpas y Seghiri (2015); Temizöz (2016); entre otros. La utilización de ítems que ya han sido utilizados en estudios anteriores puede ayudar a corroborar la calidad general del nuevo estudio, ya que son ítems que han sido evaluados previamente mediante pilotaje y diversos métodos de control y que, además, ya se han expuesto a una muestra de estudio y han podido responderse correctamente. Por todo ello, en algún caso, se prefirió reutilizar la formulación de algún ítem que aparecía en alguno de estos estudios anteriores con sus posibles respuestas, en lugar de crear otro modelo de pregunta y respuestas, cuando el tema sobre el que versaba era el mismo (por ejemplo, para conocer los años de experiencia en el sector).

En otros casos, se partió de algún ítem ya utilizado para adaptarlo a este estudio. Por ejemplo, la pregunta 16.18. («Según su opinión, ¿con quéfrecuencia considera que la persona que encarga a un traductor una posedición de textos generados con TA suele ser consciente de lo que implica esta tarea (características, dificultades, niveles de calidad, etc.)?») se adaptó a partir de otra que aparece en un cuestionario previo enfocado a la traducción inversa de Pérez-Macías (2013) («10. ¿Cree que la persona que encarga a un traductor una traducción inversa es consciente de las características y dificultades propias de esta tarea?»). Por último, se añadieron ítems de nueva creación y formulación para este estudio que, posteriormente, se sometieron a varias fases de valoración. El anexo II incluye información sobre los distintos ítems que se han tomado o que se han adaptado a partir de los estudios previos antes citados y en el anexo III se puede visualizar la versión final publicada del cuestionario.

Antes de pasar al siguiente apartado, es importante explicar los tipos de escala Likert que se han incluido en este cuestionario, que serían los siguientes: de frecuencia, de satisfacción y de probabilidad. Este tipo de escalas pueden resultar interesantes para los encuestados e incluso a veces les puede parecer entretenido completarlas (Robson, 2011: 306). En un primer momento, se dudó si incluir cuatro niveles o cinco en las respuestas de cada ítem en formato de escala, pues la inclusión de un valor central neutro tiene implicaciones importantes en el tipo de respuesta recogida. Finalmente, se decidió que sería más interesante para este estudio eliminar el valor intermedio (de indiferencia o neutralidad), con el fin de que los encuestados tuvieran que decantarse por una opción concreta (por ejemplo, en el caso de las escalas de frecuencia las opciones son: «habitualmente», «con bastante frecuencia», «con poca frecuencia»y «nunca»; además, se añade una opción «NS/NC», pero se evita poner un nivel intermedio de frecuencia porque se ha estudiado que dicha categoría puede atraer respuestas vagas o poco deliberadas (por ejemplo, Gavin, 2008: 159; Trochim, 2006). Además, al reducir el número de opciones posible se evita que haya una mayor dificultad para diferenciar entre los distintos grados de respuesta ofrecidos (Rojo, 2013). 
Por otro lado, la decisión de utilizar un cuestionario en línea se basó, fundamentalmente, en las ventajas que ofrece esta modalidad en comparación con otras opciones posibles (por ejemplo, en versión impresa). Morón (2010: 416-418) enumera las principales ventajas de este tipo de modalidad de recogida de datos, que se presentan a continuación resumidas:

- Acceso efectivo y ágil a la población de estudio.

- Familiarización de la población con este tipo de herramientas.

- Inmediatez en el contacto con los sujetos.

- Almacenamiento directo de los datos recopilados.

Robson (2011: 249), en línea con Morón, añade otras ventajas a las ya señaladas, que se presentan a continuación, traducidas y resumidas:

- Bajo coste.

- Inclusión de patrones complejos que en papel quedarían menos claros.

- Acompañamiento de ayudas como imágenes, gráficos, etc.

- Posibilidad de adaptación para personas con discapacidad visual, por ejemplo.

Fase segunda: pruebas de robustez, panel de jueces $y$ pilotaje

Con el fin de optimizar el diseño del cuestionario, es necesario que este supere una serie de pruebas de control antes de ser lanzado para evitar, entre otros, problemas de comprensión por parte de los encuestados o la inclusión de determinados sesgos (Fink, 2003: 93). Esta fase compleja ralentiza el desarrollo del estudio de campo, pero los resultados que se consiguen una vez finalizada son muy beneficiosos para el conjunto de la investigación.

En primer lugar, se estudió la validez del contenido y de la estructura del cuestionario sometiéndola al juicio externo de expertos en la materia. Morón (2010: 424) explica que con esta modalidad de pilotaje se pretende someter a evaluación de especialistas la construcción y conceptualización del instrumento, dada la reconocida experiencia de estos en metodologías de investigación en distintos campos de especialidad. Para la valoración del cuestionario, se contó con cuatro expertos en la materia ${ }^{4}$, seleccionados bien por sus conocimientos en el diseño de cuestionarios aplicados a los estudios de traducción, bien por sus conocimientos sobre posedición, o por cumplir con ambas condiciones.

Todos los jueces aprobaron el diseño del cuestionario en términos generales. No obstante, propusieron las siguientes modificaciones para optimizarlo:

- Reducir la extensión del cuestionario para evitar mortandad o pérdida de respuestas.

- Concretar y simplificar los enunciados de los ítems y las posibles respuestas.

- Solucionar casos concretos de ítems que podrían causar ambigüedad con su formulación original.

- Revisar las escalas de frecuencia, de satisfacción y de probabilidad del cuestionario para equiparar las diferentes opciones de respuestas dentro de cada uno de los tipos de escalas.

Una vez incluidas las modificaciones y sugerencias propuestas por los jueces, la nueva ver-

4 Dra. Elisa Alonso Jiménez (Universidad Pablo de Olavide, España), experta en posedición y con experiencia en el diseño de cuestionarios; Dra. Anna Kuznik (Universidad de Breslavia, Polonia), experta en el uso de cuestionarios y encuestas en el ámbito de la traducción; Dra. M. ${ }^{a}$ de los Ángeles Morón Martín (Universidad Pablo de Olavide, España), experta en el uso de cuestionarios y encuestas en el ámbito de la traducción, familiarizada con la posedición; y Dr. Lucas Nunes Vieira (University of Bristol, Reino Unido), experto en posedición. 
386

sión del cuestionario se sometió a la segunda prueba de robustez, en este caso una prueba de pilotaje. Tal y como afirma Oppenheim (1992: 49 y 55) es necesario pilotar cada uno de los componentes de un cuestionario, incluso aquellas preguntas que se hayan tomado de otros estudios previos y que ya fueron pilotadas anteriormente, puesto que podrían no funcionar de la misma manera en el contexto y con la muestra de un estudio diferente.

Con este propósito, se seleccionó a cuatro sujetos (contactos directos de la autora de la investigación) que pertenecían al público objetivo. Se les informó de que era una fase de pruebas del cuestionario para conocer su opinión y que debían completarlo y se les solicitó analizar tanto el diseño del cuestionario como el proceso de difusión, cumplimentación y registro de datos. Con el objetivo de facilitar la comunicación entre los sujetos escogidos para el pilotaje y la investigadora, se habilitó un cuadro de texto al final del cuestionario en la versión digital para que pudieran incluir los comentarios, sugerencias, etc. que consideraran oportunos respecto a la tarea realizada.

Una vez finalizado el periodo de pilotaje, se revisaron y procesaron los datos recopilados como si se tratara de los resultados reales del estudio, para comprobar así que no surgía ningún problema en la fase de su análisis. Todos los sujetos pudieron completar sin apenas incidencias el cuestionario. No obstante, uno de los sujetos indicó no haber podido realizar, en el primer intento, la encuesta al completo a través de un dispositivo móvil, debido a que había problemas de visualización de algunos ítems porque el formato utilizado para la publicación de la encuesta no estaba adaptado a dispositivos móviles, por lo que, finalmente, optó por completarla desde un ordenador, donde no tuvo ningún problema para poder terminar de rellenarla.

Con el fin de solucionar este problema, se decidió cambiar el formato del cuestionario y utilizar otro que sí estuviera adaptado a dispositivos móviles. De esta forma, todos los sujetos que quisieran cumplimentar la encuesta a través de un móvil podrían hacerlo de este modo, lo que conllevaría un mayor índice de respuestas. Una vez que se tuvo el modelo final del cuestionario, se realizó una versión en la aplicación en línea LimeSurvey.

\section{Fase tercera: lanzamiento del cuestionario virtual}

Este estudio se enmarca en un contexto de muestreo no probabilístico a través de la técnica bola de nieve, consistente en identificar varios sujetos que formen parte del público objetivo del estudio y que estos, una vez hayan participado, sean utilizados a su vez como nexo de unión para identificar a otros participantes potenciales que también formen parte del público objetivo, que deberán identificar, a su vez, a otros participantes potenciales y así sucesivamente (Oppenheim, 1992: 42-43; Robson, 2011: 275).

Con el fin de compensar el hecho de que no exista un censo donde aparezcan registrados el número total y los datos de contacto de los profesionales que pudieran pertener al público objetivo, como ya se ha explicado al comienzo del apartado 3, el cuestionario se administró fundamentalmente enviando mediante correo electrónico el enlace directamente a una lista de profesionales concretos que se habían identificado previamente y que cumplían los requisitos para poder participar. A partir del método ya descrito para el muestreo, se planificó que ellos podrían enviar el enlace a otros profesionales que cumplieran con las condiciones del perfil (este método tiene como consecuencia que no sea posible conocer el número exacto de sujetos que recibie- 
ron el cuestionario, ya que, como se puede apreciar en el diagrama 1 , conforme se va pasando de un núcleo de contactos más cercano a otro más distante, se va perdiendo el control del número de personas que reciben el cuestionario). Con este propósito, se identificaron varios núcleos de contactos que servirían como punto de partida en el método de muestreo: contactos directos (35 contactos de poseditores, de los que se descartaron los cuatro sujetos que participaron en la fase de pilotaje), empresas (aproximadamente 10 empresas de traducción que ofrecían posedición entre sus servicios), asociaciones ( $\mathrm{La}$ Xarxa Red de Traductores e Intérpretes) y, por último, redes sociales especializadas (se incluyó un mensaje pidiendo la colaboración en el estudio en cuatro grupos de Facebook formados por profesionales de la traducción: Forotraducción, con cerca de 7000 miembros (última consulta realizada el 20/03/2017); Egresados UPO Traducción e Interpretación, con unos 800 miembros (última consulta realizada el 20/03/2017); SVQ Trad, con aproximadamente $130 \mathrm{miem}$ bros (última consulta realizada el 20/03/2017); y Traductología y traducción, con alrededor de 1400 miembros (última consulta realizada el 20/03/2017). Aquí es necesario tener en cuenta que dos de los grupos de Facebook empleados favorecen que el estudio llegue a una muestra dispersa geográficamente (Forotraducción y Traductología y traducción), ya que los traductores profesionales pueden acceder a ellos con la única restricción de ser aceptados por el administrador o administradores del grupo para entrar como nuevo miembro.

Por otro lado, tanto el grupo Egresados UPO Traducción e Interpretación como el grupo SVQ Trad presentan unas características diferenciales que limitan el acceso: en el primer grupo, haber estudiado Traducción e Interpretación 387 en la Universidad Pablo de Olavide (aunque los estudiantes de hecho provienen de diferentes partes de España, especialmente de diferentes provincias de Andalucía y Extremadura), con independencia del lugar de residencia actual y, en el segundo grupo, vivir en Sevilla. Se tuvo en consideración que estas condiciones podrían dar lugar a leves sesgos de localización o de perfil de los encuestados durante el muestreo. Del mismo modo, la asociación con la que se colaboró para la distribución del cuestionario, La Xarxa, opera en la Comunidad Valenciana. Por lo tanto, en tres de los focos de lanzamiento del instrumento el entorno tendía a tener algún tipo de vinculación local, ya fuera geográfica o por perfil de cualificación, mientras que los demás eran de carácter nacional. En conjunto, se consideró que, con estos primeros puntos de contacto, con una gran mezcla de perfiles y localizaciones geográficas, la variedad de encuestados quedaba suficientemente compensada, especialmente si se tiene en cuenta que la muestra por bola de nieve se dispersa posteriormente en las siguientes difusiones y que existían otros puntos de contacto inicial como son las empresas y una lista de traductores autónomos ubicados en diferentes zonas de España (Granada, Madrid, Málaga, Barcelona, etc.), que a su vez circularon el cuestionario entre sus contactos y colaboradores. Este proceso se ha documentado mediante el siguiente gráfico representativo para comprender el proceso completo, donde se pueden identificar las fases de difusión iniciales que se pudieron planificar y controlar (en color gris).

Finalmente, el cuestionario se mantuvo activo para su realización durante dos meses (desde mediados de diciembre de 2016 a mediados de 


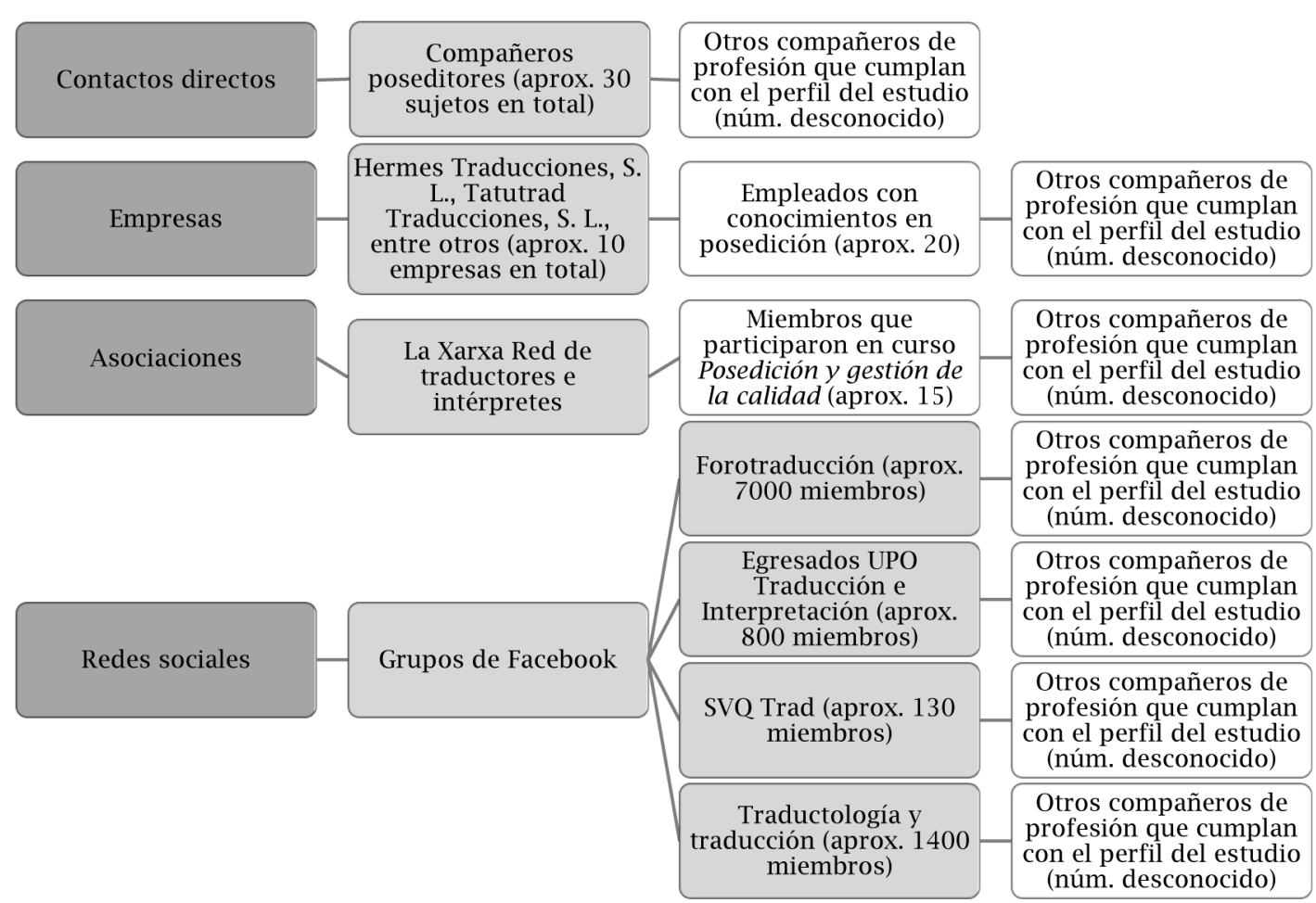

DiAgRAMA I. Sistema de muestreo mediante bola de nieve: contactos (fuente: elaboración propia)

febrero de 2017). Con el objetivo de favorecer la sinceridad y confianza de los encuestados, la participación en el cuestionario fue totalmente anónima y no se fijó un tiempo máximo para responder, incluso se incluyó una opción que permitía completarlo en varias sesiones, ya que, a través de una contraseña generada de manera automática y vinculada a la IP de acceso (para mantener el anonimato del sujeto), se podía reanudar la cumplimentación del cuestionario y continuar desde la última actualización guardada.

\section{Recogida y obtención de datos}

Como ya se ha comentado, se utilizó LimeSurvey para la publicación de la encuesta, que se presenta en su sitio web como una aplicación de código abierto para la elaboración y publicación de encuestas en línea ${ }^{5}$.

La elección del cuestionario en línea como instrumento agilizó la fase de recogida de datos, ya que las respuestas se iban guardando automáticamente conforme los participantes iban completando el cuestionario y se iba generando una base de datos en formato Excel que podía ser consultada en cualquier momento. A través del sistema utilizado para el lanzamiento de la encuesta, fue posible configurar una opción que

\footnotetext{
5 Información disponible en: <https://www.limesurvey. org>.
} 
ayudó a conocer el número de respuestas recopiladas durante todo el tiempo que estuvo activo el cuestionario, ya que se recibía un aviso en el correo electrónico cada vez que alguien pulsaba el botón «enviar resultados». Este sistema resultó muy útil para conocer el tamaño de la muestra en cada momento.

Colin Robson (2011: 413-464) dedica un capítulo completo al análisis e interpretación de datos cuantitativos, por lo que se prestó especial atención a los aspectos que pudieran resultar más relevantes para aplicarlos en nuestro estudio. El autor recuerda que el análisis de datos es un tema que hay que plantearse desde el diseño del cuestionario (Robson, 2011: 415), no solo para tener la certeza de que los datos que se van a recopilar serán posteriormente analizables, sino también para simplificar tanto como sea posible el proceso de análisis de los datos, y así se ha llevado a cabo en nuestro estudio, tal y como se ha comentado previamente en el apartado de diseño del cuestionario, donde se explican en detalle todas las decisiones tomadas para la planificación y elaboración del cuestionario.

En total, 104 individuos han participado en este estudio. Aunque se trata de una muestra que puede resultar amplia en comparación con otros estudios similares, no se puede considerar estadísticamente representativa si se tiene en cuenta la posible población total de traductores en España. Sin embargo, según la perspectiva cualitativa en la que está enmarcada este estudio, con los datos obtenidos se puede realizar un recorrido descriptivo por las percepciones actuales respecto a la posedición de los profesionales de la traducción en España.

Antes de comenzar a presentar y analizar algunos de los resultados más significativos extraídos de los datos que se han recogido mediante el cuestionario, es necesario exponer el perfil mayoritario de los traductores que participaron en este estudio. El perfil predominante entre los encuestados se corresponde con los siguientes parámetros: mujer, de entre 26 y 35 años, con español como lengua materna, con inglés como primera lengua extranjera, con la Licenciatura o el Grado en Traducción e Interpretación, para quien las actividades relacionadas con el sector de la traducción suponen su actividad principal, que realiza tareas de traducción y revisión, que trabaja por cuenta propia y que alguna vez ha realizado posedición de textos generados con traducción automática. Todos estos valores están representados entre los encuestados con más de un $60 \%$ del total.

Respecto a la experiencia profesional general de los encuestados, es importante destacar que cerca de un $90 \%$ de la muestra tenía experiencia con herramientas de traducción asistida por ordenador. En cuanto a las herramientas de traducción automática, también la mayoría tenía experiencia, aunque en un porcentaje menor, un $67 \%$. En ambos casos, la mayoría de los encuestados ha utilizado estas herramientas con fines profesionales y por iniciativa propia.

En relación con la experiencia profesional relativa a la posedición es llamativo señalar, en primer lugar, que dentro del $63 \%$ de la muestra con experiencia en posedición, la mayoría ha realizado estas tareas con fines profesionales y a petición del cliente, por lo que se podría atribuir a una situación impuesta, no como en el caso de la traducción automática o asistida por ordenador, que tiende a ser por iniciativa propia, según los participantes en el estudio. Más de la mitad de esta parte de la muestra con experiencia en posedición realiza este tipo de tareas únicamente de manera puntual, con poca frecuencia y el porcentaje respecto al total de trabajo en el último año no supondría más de un $25 \%$. La pro- 
390

ductividad media diaria parece verse incrementada con el uso de posedición, según el criterio de los encuestados.

Respecto a la opinión de los encuestados en relación con el grado de satisfacción respecto a la rentabilidad económica de los proyectos de posedición, el $42 \%$ ha indicado que el precio es poco adecuado en este tipo de tareas y el $62 \%$ señaló que cree que no es posible vivir exclusivamente de la posedición.

Para concluir, de las preguntas dirigidas a la totalidad de la muestra, independientemente de que tuvieran o no experiencia en posedición, se han obtenido también dos conclusiones muy significativas: cerca del $60 \%$ de los 104 encuestados considera la posedición como una oportunidad para los traductores, como algo positivo; sin embargo, si en una situación con condiciones de rentabilidad económica equivalentes se les preguntara qué tipo de proyecto preferirían realizar, la gran mayoría de los encuestados, el $70 \%$, escogería traducción con apoyo de traducción asistida por ordenador y la posedición quedaría relegada a un segundo puesto, muy igualada con la traducción exclusivamente humana (con un $15 \%$ y un $13 \%$ respectivamente), dato que parece volver a poner de relieve que la posedición se suele realizar cuando viene impuesta por el cliente, pero no por decisión propia.

\section{CONCLUSIONES}

Uno de los principales obstáculos que debe afrontar un investigador cualitativo del ámbito social es que su estudio pueda ser cuestionado por otros académicos que no están tan acostumbrados a este tipo de enfoque, como pueden ser los investigadores de campos relacionados con métodos experimentales (Robson, 2011). Por este motivo, cobra una mayor importancia poder demostrar la calidad del estudio que se está llevan- do a cabo y garantizar el rigor metodológico en la investigación. En el estudio que se ha presentado en esta contribución se han tenido en cuenta los siguientes aspectos para dotar de calidad a esta investigación: exhaustividad (se ha explicado en detalle el método escogido para el estudio); sistematicidad (se ha seguido una planificación previa y se han identificado y justificado los pasos dados a lo largo de la investigación); rigurosidad (se han detallado los errores detectados y se ha justificado la manera de subsanarlos); y, por último, orientación al lector (se ha tenido en mente al lector, para facilitarle el entendimiento de todo el proceso de la investigación).

Otro concepto que repercute en la idea de calidad del estudio es la validez. La triangulación metodológica llevada a cabo permite ofrecer al estudio mayor precisión y validez, ya que logra contrastar distintos tipos de datos y minimiza las posibilidades de sesgo que derivarían de una única fuente. Si nos centramos en el objetivo concreto que se planteó al comienzo de este trabajo, demostrar la validez y aplicabilidad del uso del cuestionario en un estudio sobre percepciones dentro del ámbito de la traducción profesional, parece que el uso del cuestionario en línea en el estudio social cualitativo está consolidándose paulatinamente, ya que ofrece una serie de ventajas que se han ido detallando a lo largo de este estudio, como la obtención de un gran número de información con gran rapidez y bajo coste, la garantía de participación anónima, la simplificación de la fase de recogida de datos, entre otros. Todo ello, lo convierte en un instrumento de recopilación de datos especialmente idóneo para conocer las percepciones de un determinado público objetivo, como se ha llevado a cabo en este estudio.

En cuanto a la traducción automática y la posedición, gracias a todos estos datos obtenidos en la investigación realizada, es posible valorar- 
los como unos procesos que despiertan grandes dilemas profesionales y éticos, ya que afectan a precios y rentabilidad, productividad, plazos, calidad, contexto de subordinación funcional al traducir, rol del traductor y preferencias sobre los tipos de proyectos, entre otros. Actualmente las herramientas ya no distinguen claramente entre traducción automática o asistida por ordenador y esto causa, a veces, que los traductores no sean del todo conscientes de que utilizan traducción automática. Tanto la traducción automática como la posedición son ámbitos de estudio que, con certeza, seguirán evolucionando durante los próximos años, debido al interés que suscitan entre investigadores y sectores de diversas áreas.

Los resultados que aquí se presentan podrían resultar de interés para los operadores del sector de los servicios lingüísticos, dado que pueden servir como orientación para conocer aspectos relacionados con el sector (rentabilidad, grado de implantación de esta práctica, etc.). Asimismo, las cuestiones que se refieren a la situación del sector y cómo la perciben los traductores quizás puedan servir a las asociaciones profesionales para completar la visión que emerge y el interés que suscita esta actividad relativamente nueva. Finalmente, desde la perspectiva académica, el marco metodológico del estudio puede resultar interesante para la elaboración de otras investigaciones similares de corte social cualitativo.

\section{REFERENCIAS}

Alonso, Elisa (2014): Traducción y tecnología. Análisis del uso y percepción de Wikipedia por parte de los profesionales de la traducción, Tesis doctoral, Universidad de Sevilla.

Ander-Egg, Ezequiel (1995): Técnicas de Investigación Social (24. ${ }^{a}$ edición), Buenos Aires: Lumen.

BASSEY, Michael (2005): «Three paradigms of educational research», en Andrew Pollard (ed.): Readings for
Reflective Teaching, London/NY: Continuum, 37-43.

Buendía, Leonor, M. ${ }^{a}$ Pilar Colás y Fuensanta Hernández (eds.) (1998): Métodos de investigación en Psicopedagogía, Madrid: McGraw-Hill.

Calvo, Elisa (2009): Análisis curricular de los estudios de Traducción e Interpretación en España: Perspectiva del estudiantado, Tesis doctoral, Universidad de Granada.

Calvo, Elisa (2004): «La Administración Pública ante la interpretación social: Toma de contacto en la provincia de Toledo», Puentes, 4, 7-16.

Cerezo, Beatriz (2012): La didáctica de la traducción audiovisual en España: Un estudio de caso empíricodescriptivo. Tesis doctoral, Universitat Jaume I.

FINK, Arlene (1995): The Survey Handbook, Thousand Oaks (CA): SAGE.

FINK, Arlene (2003): The Survey Kit (2nd edition), Thousand Oaks (CA): SAGE.

Gavin, Helen (2008): Understanding Research Methods and Statistics in Psychology, London: SAGE.

Golden, Sean, Amparo Hurtado y Ramón Piqué (1992): «La traducció i la interpretació a Catalunya», Noves $S L, 17,14-17$.

GonZÁLEZ, José (2001): «El paradigma interpretativo en la investigación social y educativa: nuevas respuestas para viejos interrogantes», Cuestiones pedagógicas, 15, 227-246.

Grotjahn, Rüdiger (1987): «On the Methodological Basis of Introspective Methods" en Claus Faerch y Gabriele Kasper (eds.): Introspection in Second Language Research, Bristol: Multilingual Matters.

GuERBERof, Ana (2012): Productivity and quality in the post-editing of outputs from translation memories and machine translation, Tesis doctoral, Universitat Rovira i Virgili.

Havumetsä, Nina (2012): The client factor. A study of clients' expectations regarding non-literary translators and the quality of non-literary translations. Tesis doctoral, University of Helsinki.

Hernández, Fuensanta (1998): «Diseños de investigación experimental», en Leonor Buendía, M. ${ }^{a}$ Pilar Colás y Fuensanta Hernández (eds.): Métodos de investigación en Psicopedagogía, Madrid: McGrawHill, 91-117.

Koponen, Maarit (2010): «Human Translation Evalua- 
392

tion», en SMT Workshop Gothenburg November 2, 2010. Helsinki: University of Helsinki.

KuzNiK, Anna, Amparo Hurtado y Anna Espinal (2010): «El uso de la encuesta de tipo social en Traductología. Características metodológicas», MonTI. Monografías de Traducción e Interpretación, 2, Alicante: Universitat de Valencia, 315-344.

Li, Defeng (2000): «Tailoring translation programs to social needs: a survey of professional translators», Target, 12, 127-149.

Lim, Hyang-Ok (2005): «Meeting Students' Expectations», Forum, 1, 175-204.

MackenzIE, Rosemary (2000) «POSI-tive Thinking About Quality in Translator Training in Finland», en Allison Beeby, Doris Ensinger y Marisa Presas (eds.): Investigating Translation: Selected Papers from the 4th International Congress on Translation, Barcelona, 1998, Ámsterdam: Benjamins, 213-222.

Morón, M. ${ }^{a}$ de los Ángeles (2010): Percepciones sobre el impacto de la movilidad en la formación de traductores: la experiencia de los graduados en el programa LAE (Lenguas Aplicadas Europa), Tesis doctoral, Universidad de Granada.

NidA, Eugene (1964): Toward a Science of Translating: With Special Reference to Principles and Procedures Involved in Bible Translating, Leiden: Brill.

O'BRIEN, Sharon (2002): «Teaching Post-editing: A proposal for Course Content», en 6th EAMT Workshop Teaching Machine Translation, 99-106.

Oppenheim, Abraham N. (1992): Questionnaire Design, Interviewing and Attitude Measurement, Londres: Pinter Publishers.

ORTEGA, Juan Miguel (2006): Análisis de la práctica de la interpretación judicial en España: el intérprete frente a su papel profesional, Tesis doctoral, Universidad de Granada.

PÉRez-MAcíAs, Lorena (2013): La situación actual de la traducción inversa en el mercado laboral español: estudio de campo, Trabajo de investigación inédito, Universidad Pablo de Olavide.

PÉREZ-MACíAs, Lorena (2017): Análisis de las percepciones en torno a la práctica de la posedición en el sector profesional de la traducción en España, Tesis doctoral, Universidad Pablo de Olavide.

Robson, Colin (2011): Real World Research (3rd edition), West Sussex: Wiley.
Rojo, Ana (2013): Diseños y Métodos de Investigación en Traducción (versión libro electrónico), Madrid: Editorial Síntesis.

SILVERMAN, David (2000): Doing qualitative research: $A$ practical handbook (1st edition), Thousand Oaks, CA: SAGE.

Temizöz, Özlem (2016): «Postediting machine translation output: subject-matter experts versus professional translators», Perspectives. Studies in Translation Theory and Practice, 24, 646-665.

Troснiм, William M. (2006): «The Research Methods Knowledge Base (2nd edition)» <http://www. socialresearchmethods.net/kb/> [consulta: 19-VI2018].

WAY, Catherine (2016): «The Challenges and Opportunities of Legal Translation and Translator Training in the 21st Century», International Journal of Communication 10, 1009-1029.

Wright, Kevin B. (2005): «Researching Internet-Based Populations: Advantages and Disadvantages of Online Survey Research, Online Questionnaire Authoring Software Packages, and Web Survey Services», Journal of Computer-Mediated Communication 10.

YANow, Dvora (2014): «Wherefore "Interpretive"», en Dvora Yanow y Peregrine Schwartz-Shea: Interpretation and Method. Empirical Research Methods and the Interpretive Turn (2nd edition), Londres-Nueva York: Routledge.

Youngman, Michael Brendon (1979): Analysing social and educational research data, Londres- Nueva York: McGraw-Hill Book Company.

Zaretskaya, Anna, Gloria Corpas y Miriam Seghiri (2015): «Integration of machine translation in CAT tools: state of the art, evaluation and user attitude», en SKASE Journal for Translation and Interpretation, 8, 76-88. 
1. Breve introducción sobre quién soy $y$ sobre el estudio que llevamos a cabo les aviso de que se va a grabar la entrevista y que será un feedback anónimo)

2. Presentación de los encuestados (experiencia profesional general): -Autónomo/cuenta ajena

- Agencia de traducción/clientes directos.

Actividades dentro del ámbito de la TeI traducción, localización, PE, transcreación, revisión, docencia.

- Ya dentro de la traducción, a qué campo pertenecen la mayoria de proyectos que realizan: localización, jurídico, económico, literario, cientifico, audiovisual, de todo...

- Lenguas de trabajo y direccionalidades

Años en el sector

- Via de cualificación: Licenciatura en TeI/Filologia, Máster oficial en TeI

- ¿Utilizáis o habéis utilizado alguna vez herramientas de traducción asistida por ordenador (herramientas TAO, memorias de traducción) como SDI Trados Studio, Memoo, Omegat u otras memorias de traducción similares como apoyo a vuestras traducciones? ¿En qué contexto (fines formativos, uso personal, fines empresariales...)

- ¿Utilizáis o habéis utilizado alguna vez herramientas de traducción automática (herramientas TA) como SDL Language Cloud Machine Translation, Systran, Google Translate (API) u otros motores de TA similares como apoyo a vuestras traducciones? ¿En qué contexto (fines formativos, uso personal, fines empresariales...)?

\section{Relación de los encuestados con la posedición}

¿Habéis recibido algún tipo de formación en relación con la posedición? ¿Hace cuánto tiempo? ¿Incluía algún tipo de prácticas: en empresa...?

¿Realizáis o habéis realizado alguna vez posedición de textos generados con TA? ¿En que contexto (fines formativos, uso personal, fines empresariales...)?

Contimuar con esta parte de la entrevista con los que hayan contestado esi. A los que hayan contestado "no" se les avisará más adelante para que sigan participando.

- ¿Cuánto tiempo lleváis realizando tareas de posedición de textos generados con TA?

- ¿Con qué frecuencia realizáis tareas de posedición de textos generados con TA? Expresad esa frecuencia aproximada en un porcentaje respecto al volumen total de -vuestro trabajo en el último año.

- ¿En qué lengua habéis realizado tareas de posedición? (Indicad si es A ० B/C)

- ¿Conocéis la diferencia entre posedición full o light? Explicar si la respuesta fuera «no". ¿Qué tipo de posedición os han pedido con una mayor frecuencia?

- ¿Podriais indicarnos vuestra productividad media diaria cuando poseditáis? (número de palabras/dia)

- ¿Cuál sería vuestra productividad media diaria cuando realizáis traducciones con el apoyo de herramientas TAO (SDI Trados Studio, MemoO, OmegaT ...)? ¿Y cuando realizáis las traducciones sin el apoyo de TAO ni TA (SDL Language Machine Translation, Systran, Google Translate [API] ...)? Estas dos preguntas las pueden contestar todos los encuestados, también los que no tienen experiencia con la posedición

- ¿Oué tipo de software habéis utilizado para poseditar? (gratuito, de pago, interno...)

- ¿Cómo son los plazos de entrega de una posedición de textos generados con TA en comparación con los de una traducción humana (traducción sin apoyo de TAO ni TA) del mismo número de palabras?

- Cuando trabajáis en una posedición, zcómo obtenéis normalmente ese texto generado con TA lo proporciona la agencia de traducción o el cliente, lo generáis vosotros ...?

- ¿Cuál es el motor de TA (SDL Language Cloud Machine Translation, Systran, Google Translate (API), Moses...) con el que soléis trabajar con una mayor frecuencia, cuando realizáis una posedición de textos generados con TA?

- Según vuestra experiencia, ¿cómo presupuestáis o facturáis la posedición (palabras, horas...)

- ¿Cómo ha modificado la posedición de textos generados con TA vuestra productividad con el paso del tiempo?
4. Posedicion y calidad:

Indicad, bajo vuestro propio criterio profesional, vuestro grado de satisfacción, en términos generales, con la calidad del output de texto de la TA (texto recibido para poseditar).

- ¿Con qué frecuencia habéis tenido que utilizar el texto origen como referencia al realizar una posedición de textos generados con TA?

- ¿Para qué aspectos pensáis que esta técnica de traducción (PE de TA) es más eficaz?

- Rapidez en la ejecución

Ortografia, sintaxis y puntuación

Coherencia y uniformidad terminológica

- Fluidez en el texto meta

Transferencia datos numéricos y otras variables (fechas, cifras,

Formato (cursiva negita etc)

Tablas

- Imágenes que contienen texto

Referencias o problemas culturales

Nombres propios

- LPara qué tipo de traducción creéis que la posedición de textos generados con a calidad linguistica general del producto final $Y$ en cuanto a la calidad terminolóbica del producto final?

- Creativa (audiovisual, literaria, etc)

cultural e institucional

De textos con lenguajes semicontrolados (cientificos, técnicos, etc. De textos informativos (divulgativos) - Jurídica, jurada, administrativa, etc.

En los proyectos con posedición de textos especializados (cientificos, técnicos, médicos, etc) qué proceso pensais que es más efectivo respecto a calidad general del producto final?

- Traductor profesional que posedita + traductor profesional que revisa - Traductor profesional que posedita + experto en la materia (ingeniero, médico...) que revis

Experto en la materia que posedita + traductor profesional que revisa

- Según vuestra opinión, ¿quién debe considerarse el responsable final de la
calidad de la traducción que se va a encontrar el lector de ese texto? ¿Con que frecuencia consideráis que la persona que encarga a un traductor una posedición de textos generados con TA suele ser consciente de lo qu

5. Posedición y rentabilidad económica:

- ¿Cuál es vuestro grado de satisfacción con la rentabilidad económica de los proyectos de posedición?

6. Posedición y productividad:

- ¿Consideráis que los plazos habituales para la posedición son adecuados y suficientes para conseguir una calidad satisfactoria para el cliente?

- ¿Con qué proceso pensáis que sois más productivos (es decir, con cuál os resulta más rentable económicamente vuestro tiempo de trabajo): solo tracuccín humana traducción con TAO o posedición de textos generados con

¿Consideráis que es viable vivir exclusivamente de la posedición?

7. Deontologia de la traducción respecto a la posedición

Aquí ya pueden contestar todos, hayan o no realizado posedición alguna vez.

- Completad la siguiente afirmación: "El cada vez más frecuente uso de la posedición de textos generados con TA en el ambito de la traducción frente la traducción humana es, según su opinión..) (oportunidad/amenaza para el

- Según vuestra opinión, zque grado de dificultad conlleva el proceso de posedición de textos generados con TA en comparación con el de traducción

- Si os dieran a elegir, zqué tipo de proyecto prefeririais asumir suponiendo que la rentabilidad economica por hora fuera equivalente en cualquiera de las generados con TA?

- Si el cliente no os pide expresamente que traduzcáis usando para ello la posedición de textos generados con TA, ¿creéis que deberiais informarle si utilizara motu proprio esta modalidad en un encargo?

8. Cierre de la entrevista y agradecimiento.

- Pueden añadir cualquier comentario respecto a todo lo que se ha tratado en la entrevista

Agradezco la colaboración de todos los entrevistados y el interés mostrado por Agradezco the 


\section{ANEXO II. PROCEDENCIA DE LOS ÍTEMS QUE NO SON DE NUEVA CREACIÓN}

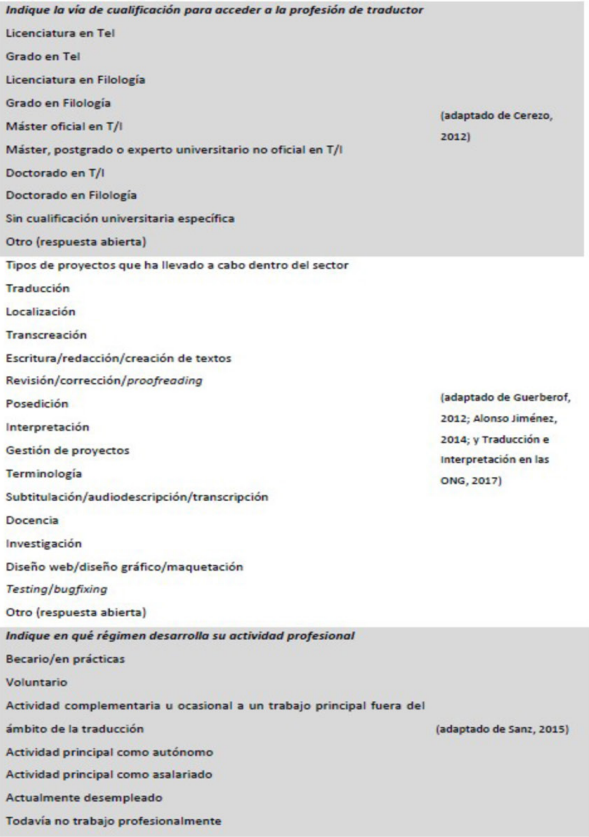

¿Cuál es su situación laboral?

Trabajo por cuenta propia y gestiono mi propia empresa o agencia del sector de la traducción

Trabajo por cuenta propia y ofrezco mis servicios como autónomo principalmente a empresas o agencias del sector de la traducción

Trabajo por cuenta propia y ofrezco mis servicios como autónomo principalmente a clientes directos ajenos al sector de la traducción Trabajo por cuenta ajena en una empresa proveedora de servicios lingüisticos del sector de la traducción

Trabajo por cuenta ajena en una empresa no perteneciente al sector de fiménez, 2014) la traducción

Trabajo por cuenta ajena en una institución pública (regional, estatal, internacional)

No recibo remuneración económica por mi labor dentro del sector de la traducción (prácticas, voluntariado)

Estoy desempleado

Estoy desempleado y realizando un curso de formación

Otro (respuesta abierta)

Años de experiencia

Menos de 1 año

De 1 a 2 años

De 3 a 5 años

De 6 a 10 años

Itomado de Guerberof,

De 11 a 15 años

2012)

\begin{tabular}{|c|c|}
\hline $\begin{array}{l}\text { ¿De qué tipo era esa formación recibida? } \\
\text { Autodidacta (lecturas, práctica profesional, aprendizaje de } \\
\text { compañeros) }\end{array}$ & \multirow{9}{*}{$\begin{array}{l}\text { (adaptado de cerezo, } \\
\text { 2012) }\end{array}$} \\
\hline Formación interna en empresas & \\
\hline Asistencia a talleres, congresos, jornadas, conferencias, etc. & \\
\hline Cursos online (MOOC, webinar, etc) & \\
\hline $\begin{array}{l}\text { Cursos universitarios de especialización, actualización y formación } \\
\text { continua o permanente }\end{array}$ & \\
\hline Asignaturas de licenciatura/grado & \\
\hline Asignaturas de máster o postgrado especializado en PE & \\
\hline Asignaturas de máster o posterado no especifico en $\mathrm{PE}$ & \\
\hline Otro (respuesta abierta) & \\
\hline \multicolumn{2}{|l|}{ ¿Ha realizado prácticas en empresa relacionadas con PE? } \\
\hline si & ladaptado de Cerezo, \\
\hline No & 2012) \\
\hline \multicolumn{2}{|l|}{$\mathrm{NS} / \mathrm{NC}$} \\
\hline \multicolumn{2}{|l|}{ ¿Qué duración tuvieron? } \\
\hline \multicolumn{2}{|l|}{ Menos de 1 mes } \\
\hline De 1 a 3 meses & 2012) \\
\hline \multicolumn{2}{|l|}{ De 4 a 6 meses } \\
\hline \multicolumn{2}{|l|}{ Más de 6 meses } \\
\hline \multicolumn{2}{|l|}{ ¿En qué contexto ha utilizado esas herramientas de TAO? } \\
\hline \multicolumn{2}{|l|}{$\begin{array}{l}\text { Con fines formativos, de investigación o realización de prácticas para } \\
\text { entender su funcionamiento }\end{array}$} \\
\hline $\begin{array}{l}\text { Con fines profesionales por iniciativa propia (creación de memorias de } \\
\text { traducción para futuros proyectos, etc.) }\end{array}$ & (adaptado de Zaretskaya \\
\hline $\begin{array}{l}\text { traduccion para futuros provectos, etc.) } \\
\text { Con fines profesionales a petición del cliente }\end{array}$ & \\
\hline $\mathrm{Ns} / \mathrm{NC}$ & \\
\hline Otro (respuesta abierta) & \\
\hline
\end{tabular}

\begin{tabular}{|c|c|}
\hline \multicolumn{2}{|l|}{ ¿En qué contexto ha utilizado esas herramientas de TA? } \\
\hline \multirow{2}{*}{\multicolumn{2}{|c|}{$\begin{array}{l}\text { Con fines formativos, de investigación o realización de prácticas para } \\
\text { entender su funcionamiento }\end{array}$}} \\
\hline & \\
\hline \multicolumn{2}{|l|}{$\begin{array}{l}\text { Con fines de uso estrictamente personal por iniciativa propia } \\
\text { (traducción automática de correo electrónico personal, redes sociales, }\end{array}$} \\
\hline & ladaptado de Zaretskaya \\
\hline Con fines profesionales por iniciativa propia (primer borrador de un & al, 2015) \\
\hline \multicolumn{2}{|l|}{ encargo, evaluación previa de dificultad, etc.) } \\
\hline \\
\hline \multirow{2}{*}{\multicolumn{2}{|c|}{$\begin{array}{l}\text { encargo, evalua ción previa de dificultad, etc.) } \\
\text { NS/NC }\end{array}$}} \\
\hline & \\
\hline \multicolumn{2}{|l|}{ Otro (respuesta abierta) } \\
\hline \multicolumn{2}{|l|}{ ¿Tiene experiencia en PE? } \\
\hline si & (adaptado de Temizōzz, \\
\hline No & 2016) \\
\hline \multicolumn{2}{|l|}{ NS/NC } \\
\hline \multicolumn{2}{|l|}{ ¿Con qué frecuencia realiza tareas de PE? } \\
\hline \multicolumn{2}{|l|}{$\begin{array}{l}\text { Habitualmente, forman parte de mis servicios habituales como } \\
\text { traductor }\end{array}$} \\
\hline $\begin{array}{l}\text { Con bastante frecuencia, aunque no forman parte de mis servicios } \\
\text { habituales como traductor }\end{array}$ & (adaptado de pérez, 2013) \\
\hline \multicolumn{2}{|l|}{ Con poca frecuencia, solo de manera puntual (compromiso, ajuste de } \\
\hline \multicolumn{2}{|l|}{ carga de trabajo, etc.) } \\
\hline \multicolumn{2}{|l|}{ NS/NC } \\
\hline \multicolumn{2}{|l|}{ Indique el porcentaje que supone la PE de TA en el volumen total de su } \\
\hline \multicolumn{2}{|l|}{ trabajo } \\
\hline \multicolumn{2}{|l|}{ De 76 a $100 \%$} \\
\hline De 51 a $75 \%$ & (adaptado de Guerberof, \\
\hline De 26 a $50 \%$ & 2012) \\
\hline De 0 a $25 \%$ & \\
\hline NS/NC & \\
\hline
\end{tabular}




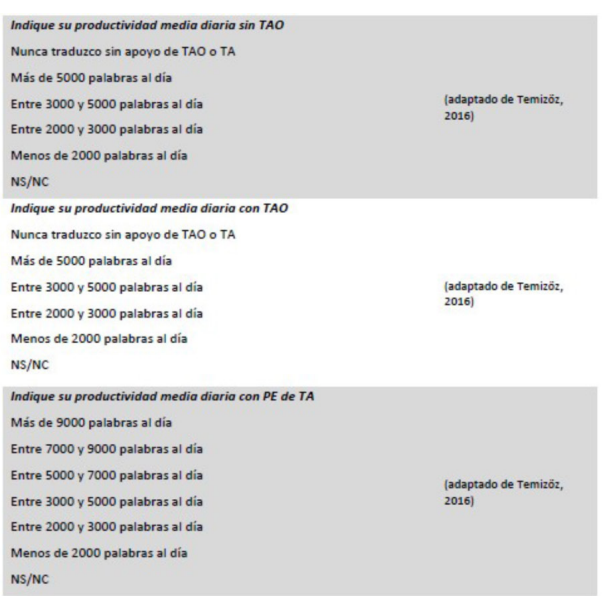

\section{¿Con qué frecuencia es posible acceder al texto original?}

Siempre he tenido acceso al texto original

Con bastante frecuencia, he podido acceder al texto original cuando lo he necesitado

Con poca frecuencia, solo en casos excepcionales

Nunca he tenido acceso al texto original

NS/NC

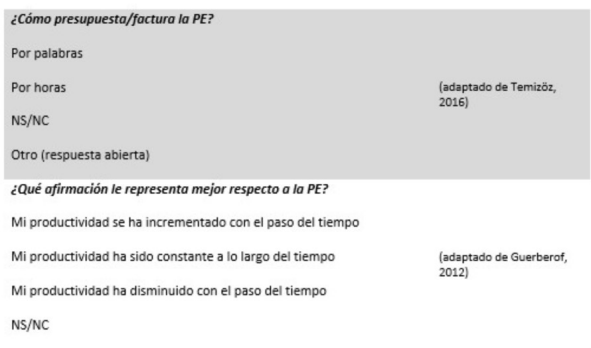

(adaptado de Temizöz, 2016)
¿Quién debe considerarse el responsable final de la calidad de la traducción?

El cliente que demanda la PE

La empresa proveedora de servicios lingüisticos que ofrece la PE

El traductor que postedita

(adaptado de Havumetsā,

2012)

NS/NC

Otro (respuesta abierta)

¿Con qué frecuencia considera que la persona que encarga $a$ un traductor una PE de TA suele ser consciente de lo que implica esto tarea?

Siempre

Con bastante frecuencia

[adaptado de Pérez, 2013)

Con poca frecuencia

Nunca

NS/NC 


\section{ANEXO III. VERSIÓN FINAL PUBLICADA DEL CUESTIONARIO ${ }^{6}$}

\section{INFORMACIÓN PARA LOS PARTICIPANTES}

El siguiente cuestionario se ha diseñado para conocer la percepción de la práctica de la posedición enel sector profesional de la traducción en Espania. Por ello, se dirige a profesionales de la traducción que cumplan, al menos, uno de los siguientes requisitos:

1) Tener experienciaen proyectos de posedición de textos generados con traducción automática.

2) Haber recibido formación en posedición de textos generados con traducción automática.

3) Considerarse familiarizado con los servicios de posedición de textos generados con traducción automática.

Completar este cuestionario no le llevará más de 15 minutos. Si no tuviera tiempo para finalizarlo en una sola sesión, el sistema guardará los resultados y podrá continuar respondiendo donde lo dejó cuando vuelva a acceder. Es totalmente anónimo y confidencial, ylos datos sobre su perfli profesional serán utilizados únicamente con fines estadisticos.

La investigadora responsable de este estudio es Lorena Pérez Macias, bajo ladirección de la Dra. Elisa Calvo, yse desarrolla en el marco de una tesis del programa de Doctorado "lenguas Modernas Traducción y Espaniol como Lengua Extranjera" de la Universidad Pablo de Olavide, de Sevilla. ¡Muchas gracias por sucolaboración!

Hay 46 preguntas en estaencuesta.

Perfil del encuestado

\section{A) PERFILDEL ENCUESTADO}

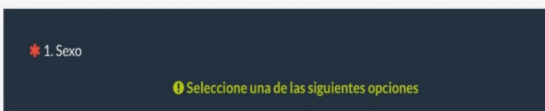

\section{Hombre}

Mujer

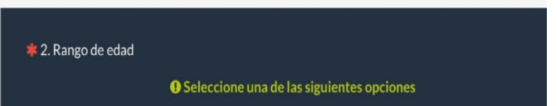

$\begin{array}{ll}\text { Menos de } 25025 & \text { De } 36 \text { a } 40 \\ \text { De 26a } 30 & \text { De 40a } 45 \\ \text { De } 31 \text { a } 35 & \text { Más de } 45\end{array}$

\section{O Marque las opciones que correspondan}

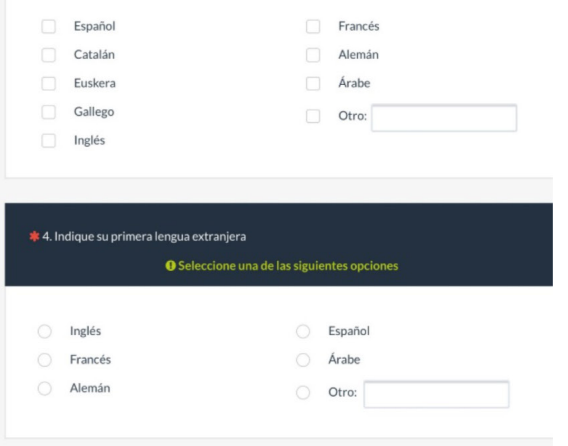

* 5. Indique su segunda lengua extranjera

O Seleccione una de las siguientes opciones

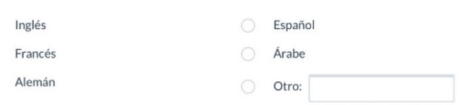

6. En el caso de que las tuviera, indique otras lenguas con las que trabaje
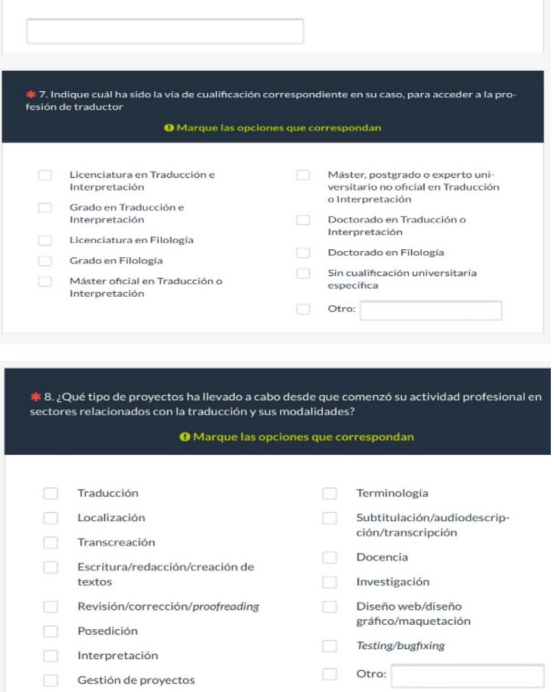

Terminologia

Docencia

Diseño web/diseño

Testing/bugfixing

Otro:

${ }^{6}$ La versión final del cuestionario (inactivo actualmente) se puede consultar en el siguiente enlace: $<$ https://goo.gl/doQYAJ>. 

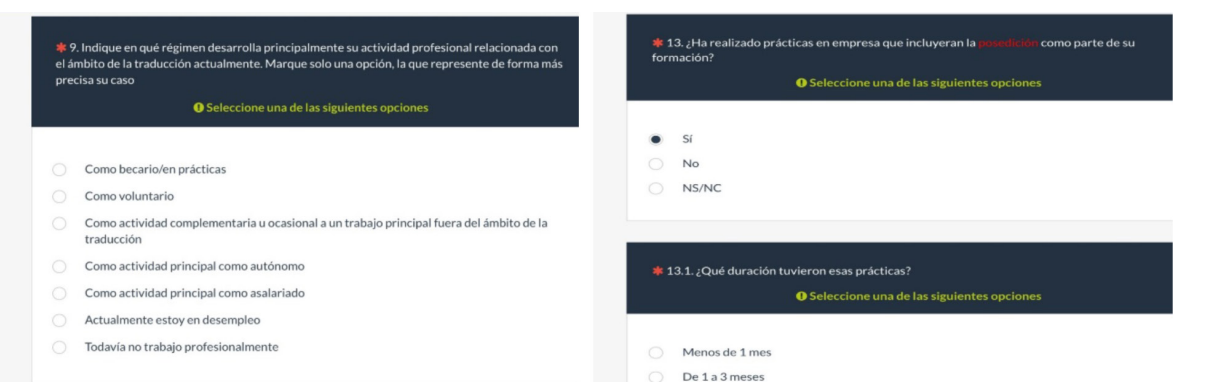

*10. Cual d de los siguientes enunciados describe mejor su situación Ilaboral? Marque solo la op.
cion predominante - Selecclone una de las siguilentes opcliones

Trabajo por cuenta propia y gestiono mi propia empresa o agencia del sector de

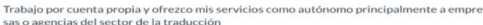

Trabajo por cuenta propia y ofrezco mis servicios como autónomo principalmente a clientes

Trabajo por cuenta ajena en una empresa proveedora de servicios lingǘsticos del sector de

Trabajo por cuenta ajena en una empresa no perteneciente al sector de la traducción

Trabajo por cuenta ajena en una institución pública (regional, estatal, internacional)

No recibo remuneración económica por mi labor dentro del sector de la traducción (prácticas, voluntariado)

Estoy desempleado

Estoy desempleado y realizando un curso de formacion

Mi situacion laboral es distinta de las enunciadas con anterioridad (indique cual)

\section{* 11. Indique los anos de experiencia en el sector
\[ \text { O Seleccione una de las siguientes opciones } \]}

Menos de 1 año

De 1 a 2 años

De 3 a 5 años

De 6 a 10 años

De 11 a 15 anos

Más de 15 anos

No tengo experiencia

* 12. ¿Ha recibida algùn tipo de formación en relación con la o Seleccione una de las siguientes opciones

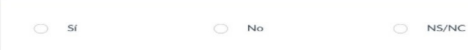

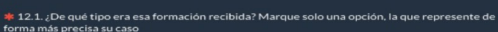
o seleccione una de las siguientes opciones

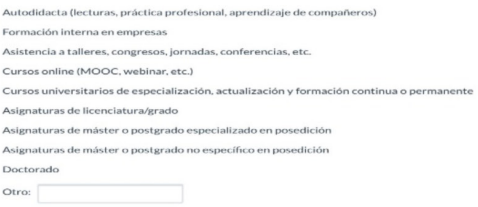

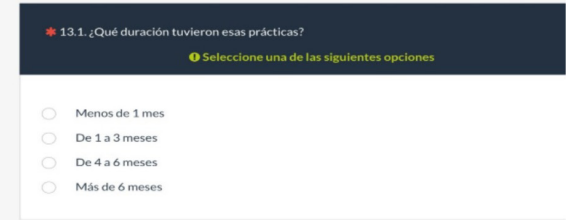

Experiencia Profesional General

B) EXPERIENCIA PROFESIONAL GENERAL

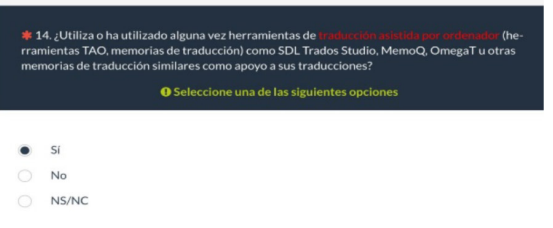

* 14.1. ¿En qué contexto utiliza o ha utilizado esas herramientas TAO?

- Marque las opciones que correspondan

Con fines formativos, de investigación o realización de prácticas para entender su uncionamiento

Con fines profesionales por iniciativa propia (creación de memorias de traducción para fu(uros proyectos, etc)

Con fines profesionales a petición del cliente

NS/NC

Otro:

* 15. ¿Utiliza o ha utllizado alguna vez herramientas de : (herramientas TA) como SDL Language Cloud Machine Translation, 5 stras
tores de TA similares como apoyo a sus traducciones? - Seleccione una de las siguientes opciones

-

No

NS/NC

* 15.1. ¿En qué contexto utiliza o ha utilizado esas herramientas TA? - Marque las opciones que correspondan

Con fines formativos de investigación o realización de prácticas para entender funcionamiento

Con fines de uso estrictamente personal por iniciativa propia (traduccion automática de orreo electrónico personal, redes sociales, etc.

Con fines profesionales por iniciativa propia (primer borrador de un encargo. evaluación previa de dificultad, etc.)

Con fines profesionales a petición del cliente (primer horrador de un encargo, evaluacion previa de dificultad, etc.)

NS/NC

Otro: 
Experiencia profesional respecto a la posedición

C) EXPERIENCIAPROFESIONAL RESPECTOALA POSEDICION

*16. iRealiza o ha realizado alguna vez

- Seleccione una de las sizuientes opciones

- si

No

NS/NC

* 16.1. ¿En qué contexto realiza o ha realizado posedición de textos generados con TA? o Marque las opciones que correspondan

Con fines formativos, de investigación o realización de prácticas para entender su

Con fines de uso estrictamente personal por iniciativa propia (posedición de correo elec.

Con fines profesionales por iniciativa propia (correo electrónico profesional, publicaciones

con caräcter profesional en redes sociales, blog, página web profesional, etc.)

- Con fines profesionales a petición del cliente (realización de encargo de posedición)

NS/NC

Otro:

* 16.2. ¿Con qué frecuencia realiza tareas de posedición de textos generados con TA?

- Seleccione una de las siguientes opciones

- Habitualmente, forman parte de mis servicios habituales como traductor

Con bastante frecuencia, aunque no forman parte de mis servicios habituales como traductor

Con poca frecuencia, solo de manera puntual (compromiso, ajuste de carga de trabajo, etc) NS/NC

* 16.3. Indique el porcentaje aproximado, de media, que ha supuesto la posedición de textos generados con TA en el volumen total de su trabajo

- Seleccione una de las siguientes opciones

- De 76 a $100 \%$

De 51 a $75 \%$

De 26 a $50 \%$

De 0 a $25 \%$

NS/NC

* 16.4. ¿En qué lengua ha realizado tareas de posedición?

- Seleccione una de las siguientes opciones

Exclusivamente en mi lengua materna

Exclusivamente en una lengua extranjera

Tanto en mi lengua materna como en la extranjera, aunque más veces en la materna Tanto en mi lengua materna como en la extranjera, aunque más veces en la extranjera Tanto en mi lengua materna como en la lengua extranjera, en la misma proporción NS/NC

* 16.5. ¿Cuánto tiempo lleva realizando tareas de posedición de textos generados con TA - Seleccione una de las siguientes opciones
16.6. ¿Qué tipo de posedición de textos generados con TA le demandan con mayor frecuencia? - Seleccione una de las siguientes opciones

- Posedición parcial (también denominada light); solo se requiere un texto que sea comprensible en la otra lengua, uso interno o informativo

Posedición completa (también denominada full): texto revisado de alta calidad y valido para publicar

NS/NC

\section{* 16.7. Indique su productividad media diaria aproximada al traducir}

Go (SDL Tra dote [API]_.

- Seleccione una de las siguientes opciones

Nunca traduzco sin apoyo de TAO $\circ$ TA

Más de 5000 palabras al día

Entre 3000 y 5000 palabras al día

Entre 2000 y 3000 palabras al dia

Menos de 2000 palabras al día

NS/NC

* 16.8. Indique su productividad media diaria aproximada al traducir

Nunca traduzco con apoyo de TAO

Más de 5000 palabras al día

Entre 3000 y 5000 palabras al dis

Entre 2000 y 3000 palabras al dia

Menos de 2000 palabras al dia

NS/NC

* 16.9. Indique su productividad media diaria

- Seleccione una de las siguientes opciones
Más de 9000 palabras/diá
Entre 7000 y 9000 palabras/día
Entre 5000 y 7000 palabras/dia
Entre 3000 y 5000 palabras/dia
Entre 2000 y 3000 palabras/día
Menos de 2000 palabras/día
NS/NC

* 16.10. Al poseditar, zqué motor de TA es el más frecuentemente empleado para generar las traducciones automáticas con las que trabaja?

\section{- Seleccione una de las siguientes opciones}

- SDL Language Cloud Machine Translation

Systran

Google Translate (API)

Moses

PROMT

NS/NC

Otro:

Más de 8 años

De 6 a 8 años

De 4 a 5 años

De 2 a 3 años

Menos de 1 ano

NS/NC 

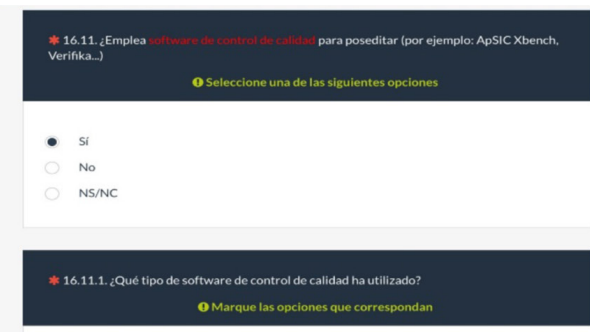

Software gratuito, ya sea software libre o de código cerrado (por ejemplo: ApSIC Xbencho MateCat)

Software de pago (por ejemplo: MemoQ o Verifika)

Software proporcionado por la empresa en la que trabajo o por el cliente (software

interno

NS/NC

Otro:

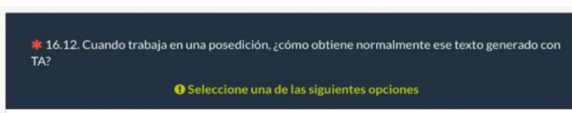

- Lo proporciona la agencia de traducción o el cliente

Lo genero yo a través de alguna herramienta de TA gratuita o de pago

$\mathrm{NS} / \mathrm{NC}$

Otro:

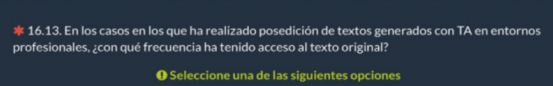

- Siempre he tenido acceso al texto original

Con bastante frecuencia, he podido acceder al texto original cuando lo he necesitado

Con poca frecuencia, solo en casos excepcionales

Nunca he tenido acceso al texto original

NS/NC

\section{* 16.14. Según su experiencia, ¿cómo presupuesta o factura la posedición?} - Seleccione una de las siguientes opciones
Por palabras
Porhoras
NS/NC
Otro:

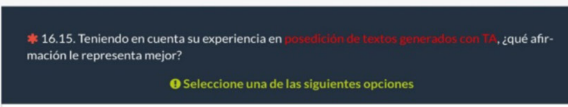

Mi productividad se ha incrementado con el pasodel tiempo

Mi productividad ha sido constante a lo largo del tiempo

Mi productividad ha disminuido con el paso del tiempo

NS/NC
Opinión personal sobre la práctica de la posedición (desde la experiencia)

D) OPINIÓN PERSONAL SOBRE LA PRACTICA DE LA POSEDICION (DESDE LA EXPERIENCIA)

\section{POSEDICION Y CALIDAD}

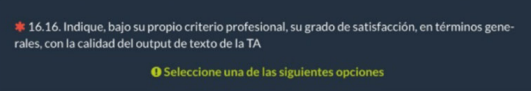
Muy satisfecho (output de muy buena calidad)
Bastante satisfecho (output de buena calidad)
Poco satisfecho (output de calidad suficiente)
Nada satisfecho (output de mala calidad)
NS/NC
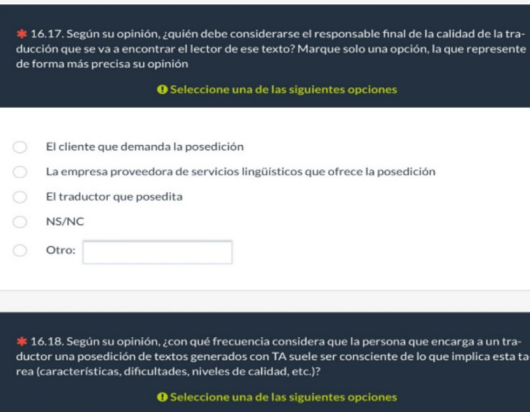

Siempre

Con bastante frecuencia

Con poca frecuencia

Nunca

NS/NC

POSEDICIÓN YRENTABILIDAD ECONÓMICA

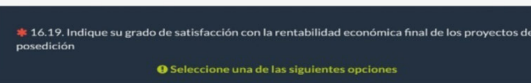

- Muy satisfecho (precio muy adecuado) Bastante satisfecho (precio bastante adecuado)

Poco satisfecho (precio poco adecuado) Nada satisfecho (precio nada adecuado) NS/NC

POSEDICIONY PRODUCTIVIDAD

* 16.20. zConsidera que los plazos habituales para la posedicion son adecuados y suncientes para conseguir una calidad sa - Seleccione una de las siguientes opciones

- Si No $\quad$ NS/NC 
* 16.21. ¿Con cuál de estos procesos piensa que es más productivo (es decir, con cuál le resulta más rentable económicamente su tiempo de trabajo)? Marque solo una opción, la que represente de forma más precisa su caso

- Seleccione una de las siguientes opciones

- Solo traducción humana (traducción sin apoyo de TAO ni TA)

Traducción con TAO

Posedición de textos generados con TA

NS/NC

Otro:

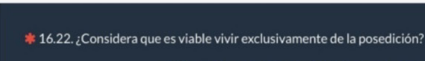

- Seleccione una de las siguientes opciones

- $\mathrm{Si}$

No

NS/NC

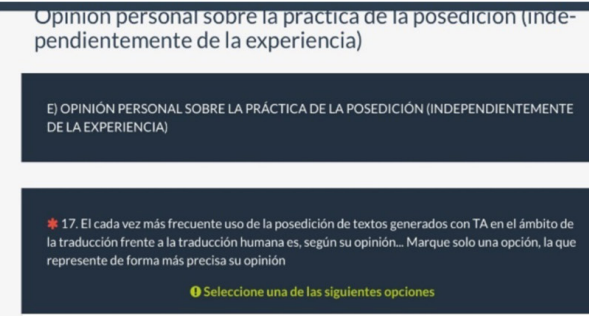

Una oportunidad para los traductores

Una amenaza para los traductores

NS/NC

* 18. Si le dieran a elegir, iqué tipo de provecto preferiria aceptar, suponiendo que la rentabilidad económica fuera equivalente en cualquiera de las opciones? Marque solo una opción, la que represente de forma más precisa su opinión

\section{O Seleccione una de las siguientes opciones}

Solo traducción humana (traducción sin apoyo de TAO ni TA)

Traducción con apoyo de TAO

Posedición de textos generados con TA

NS/NC
* 19. Si el cliente no le pide expresamente que traduzca con posedición de textos generados con

$\mathrm{TA}$, ¿cree que deberia informarle si utilizara motu proprio esta modalidad en un encargo? - Seleccione una de las siguientes opciones

- Si, es necesario en cualquier caso

Si, es necesario, pero solo si el resultado de la posedición va a constituir la base de la entrega final del proyecto

No es necesario si el resultado de la posedición es solo a titulo personal como primera aproximación al proyecto y no va a constituir la base de la entrega final del proyecto

No, en ningún caso, es una decisión del traductor exclusivamente

NS/NC 